\title{
Dynamical analysis of a biological resource management model with impulsive releasing and harvesting
}

\author{
Jianjun Jiao ${ }^{1 *}$, Lansun Chen ${ }^{2}$ and Shaohong Cai ${ }^{1}$
}

\author{
* Correspondence: \\ jiaojianjun05@126.com \\ ${ }^{1}$ School of Mathematics and \\ Statistics, Guizhou Key Laboratory \\ of Economic System Simulation, \\ Guizhou University of Finance and \\ Economics, 550004 Guiyang, P. R. \\ China \\ Full list of author information is \\ available at the end of the article
}

\begin{abstract}
In this study, we consider a biological resource management predator-prey model with impulsive releasing and harvesting at different moments. First, we prove that all solutions of the investigated system are uniformly ultimately bounded. Second, the conditions of the globally asymptotic stability predator-extinction boundary periodic solution are obtained. Third, the permanence condition of the investigated system is also obtained. Finally, the numerical simulation verifies our results. These results provide reliable tactic basis for the biological resource management in practice.
\end{abstract}

Keywords: predator-prey model, impulsive releasing, impulsive harvesting, extinction, permanence

\section{Introduction}

Biological resources are renewable resources. Economic and biological aspects of renewable resources management have been considered by Clark [1]. In recent years, the optimal management of renewable resources, which has direct relationship to sustainable development, has been studied extensively by many authors [2-4]. Especially, the predator-prey models with harvesting (or dispersal and competition) are investigated by many articles [5-8]. In general, the exploitation of population should be determined by the economic and biological value of the population. It is the purpose of this article to analyze the exploitation of the predator-prey model with impulsive releasing and harvesting at different moments.

Impulsive delay differential equations are suitable for the mathematical simulation of the evolutionary process. The application of impulsive delay differential equations to population dynamics is an interesting topic since it is reasonable and correct in modelling the evolution of population, such as pest management [9]. Moreover, impulsive delay differential equations are used in various fields of applied sciences too, for example physics, ecology, pest control and so on. According to the nature of biological resource management, Jiao et al. [10] introduced the stocking on prey at fixed moments, and considering the following impulsive delay differential equation 


$$
\left\{\begin{array}{l}
x^{\prime}{ }_{1}(t)=x_{1}(t)\left(a-b x_{1}(t)\right)-\frac{\beta x_{1}(t)}{1+c x_{1}(t)} x_{3}(t), \\
x^{\prime}{ }_{2}(t)=r x_{3}(t)-r e^{-w \tau_{1}} x_{3}\left(t-\tau_{1}\right)-w x_{2}(t), \\
x^{\prime}{ }_{3}(t)=r e^{-w \tau_{1}} x_{3}\left(t-\tau_{1}\right)+\frac{k \beta x_{1}(t)}{1+c x_{1}(t)} x_{3}(t)-d_{3} x_{3}(t)-E x_{3}(t)-d_{4} x_{3}^{2}(t), \\
\Delta x_{1}(t)=\mu, \\
\Delta x_{2}(t)=0, \\
\Delta x_{3}(t)=0, \\
\left(\varphi_{1}(\zeta), \varphi_{2}(\zeta), \varphi_{3}(\zeta)\right) \in C_{+}=C\left(\left[-\tau_{1}, 0\right], R_{+}^{3}\right), \quad \varphi_{i}(0)>0, \quad i=1,2,3 .
\end{array} t \neq n \tau,\right.
$$

The biological meanings of the parameters in (1.1) can be seen in [10]. Jiao and Chen [10] consider the mature predator population is harvested continuously. In fact, the population with economic value are harvested discontinuously. It will be arisen at fixed moments or state-dependent moments, that is to say, the releasing population and harvesting population should be occurred at differential moments in [10]. In this article, in order to model the fact of the biological resource management, we investigate a differential equation with two impulses for the biological resource management.

\section{The model}

It is well known that the basic Lotka-Volterra predator-prey model can be written as

$$
\left\{\begin{array}{l}
\frac{\mathrm{d} x_{1}(t)}{\mathrm{d} t}=x_{1}(t)\left(r-a x_{1}-b x_{2}(t)\right), \\
\frac{\mathrm{d} x_{2}(t)}{\mathrm{d} t}=x_{2}(t)\left(-d+c x_{1}(t)\right),
\end{array}\right.
$$

where $x_{1}(t)$ and $x_{2}(t)$ are densities of the prey population and the predator population, respectively, $r>0$ is the intrinsic growth rate of prey, $a>0$ is the coefficient of intraspecific competition, $b>0$ is the per-capita rate of predation of the predator, $d>0$ is the death rate of predator, $c>0$ denotes the product of the per-capita rate of predation and the rate of conversing prey into predator. If $r c<a d$ is satisfied, the predator $x_{2}(t)$ will go extinct and the prey will tend to $r / a$, that is to say, system $(2.1)$ has boundary equilibrium $r / a, 0$ ). If $r c>a d$ is satisfied, system (2.1) has globally asymptotically stable unique positive equilibrium $(d / c, r c-a d / c b)$.

System (2.1) is an organic growth model, that is to say, there is no intervention management on system (2.1). Obviously, the dynamical behaviors of system (2.1) is very simple. As a matter of fact, the mankind more and more devote themselves to investigate and empolder the ecosystem with the development of society. Bases on the ideology, we develop (2.1) by introducing releasing the prey and harvesting the predator and prey at different fixed moments, that is, we consider the following impulsive differential equation

$$
\left\{\begin{array}{l}
\frac{\mathrm{d} x(t)}{\mathrm{d} t}=x(t)(a-b x(t))-\beta x(t) y(t), \\
\frac{\mathrm{d} y(t)}{\mathrm{d} t}=k \beta x(t) y(t)-\mathrm{d} y(t), \\
\left.\begin{array}{c}
\Delta x(t)=-\mu_{1} x(t), \\
\Delta y(t)=-\mu_{2} \gamma(t),
\end{array}\right\} \quad t=(n+l) \tau, \quad n=1,2, \ldots \\
\left.\begin{array}{rl}
\Delta x(t) & =\mu, \\
\Delta y(t) & =0,
\end{array}\right\} \quad t=(n+1) \tau, \quad n=1,2, \ldots
\end{array}\right.
$$


where $x(t)$ denotes the density of the predator population at time $t . y(t)$ denotes the density of the prey population $Y$ at time $t . a>0$ denotes the intrinsic growth rate of the prey population $X . b>0$ denotes the coefficient of the intraspecific competition in prey population $X . \beta>0$ denotes the per-capita rate predation of the predator population $Y . k>0$ denotes product of the per-capita rate and the rate of conversing prey population $X$ into predator population $Y . d>0$ denotes the death rate of the predator population $Y .0<\mu_{1}<1$ denotes the harvesting rate of prey population $X$ at $t=(n+$ l) $\tau, n \in Z^{+} .0<\mu_{2}<1$ denotes the harvesting rate of predator population $Y$ at $t=(n+$ l) $\tau, n \in Z^{+} . \mu>0$ denotes the released amount of prey population $X$ at $t=(n+1) \tau, n$ $\in Z^{+} . \Delta x(t)=x\left(t^{+}\right)-x(t)$, where $x\left(t^{+}\right)$represents the density of prey population $X$ immediately after the impulsive releasing (or harvesting) at time $t$, while $x(t)$ represents the density of prey population $X$ before the impulsive releasing (or harvesting) at time t. $\Delta y(t)=y\left(t^{+}\right)-y(t)$, where $y\left(t^{+}\right)$represents the density of predator population $Y$ immediately after the impulsive harvesting at time $t$, while $y(t)$ represents the density of predator population $Y$ before the impulsive harvesting at time $t .0<l<1$, and $\tau$ denotes the period of impulsive effect.

\section{The lemmas}

Before discussing main results, we will give some definitions, notations and lemmas. Let $R_{+}=[0, \infty), R_{+}^{2}=\left\{z \in R^{2}: z>0\right\}$. Denote $f=\left(f_{1}, f_{2}\right)$ the map defined by the right hand of system (2.2). Let $V: R_{+} \times R_{+}^{2} \rightarrow R_{+}$, then $V$ is said to belong to class $V_{0}$, if

(i) $V$ is continuous in $(n \tau,(n+l) \tau] \times R_{+}^{2}$ and $((n+l) \tau,(n+1) \tau] \times R_{+}^{2}$, for each $\lim _{(t, y) \rightarrow\left(n \tau^{+}, z\right)} V(t, z)=V\left(n \tau^{+}, z\right), \quad \quad \lim _{(t, y) \rightarrow\left(n \tau^{+}, z\right)} V(t, z)=V\left(n \tau^{+}, z\right) \quad$ and $\lim _{(t, z) \rightarrow\left((n+l) \tau^{+}, z\right)} V(t, z)=V\left((n+l) \tau^{+}, z\right)$ exists.

(ii) $V$ is locally Lipschitzian in $z$.

Definition 3.1. $V \in V_{0}$, then for $(t, z) \in(n \tau,(n+l) \tau] \times R_{+}^{2} \quad$ and $((n+l) \tau,(n+1) \tau] \times R_{+}^{2}$, the upper right derivative of $V(t, z)$ with respect to the impulsive differential system (2.2) is defined as

$$
D^{+} V(t, z)=\lim _{h \rightarrow 0} \sup \frac{1}{h}[V(t+h, z+h f(t, z))-V(t, z)] .
$$

The solution of system (2.2), denote by $z(t)=(x(t), y(t))^{T}$, is a piecewise continuous function $\quad z: R_{+} \rightarrow R_{+}^{2}, \quad z(t) \quad$ is continuous on $(n \tau,(n+l) \tau] \times R_{+}^{2}$ and $((n+l) \tau,(n+1) \tau] \times R_{+}^{2}\left(n \in Z_{+}, 0 \leq l \leq 1\right)$. Obviously, the global existence and uniqueness of solutions of (2.2) is guaranteed by the smoothness properties of $f$, which denotes the mapping defined by right-side of system (2.2) (see Lakshmikantham [4]).

Before we have the main results. We need give some lemmas which will be used in the next. Since $(\mathrm{d} x(t) / \mathrm{d} t=0)$ whenever $x(t)=0, \mathrm{~d} y(t) / \mathrm{d} t=0$ whenever $y(t)=0, t=n \tau$, $x\left(n \tau^{+}\right)=\left(1-\mu_{1}\right) x(n \tau), y\left(n \tau^{+}\right)=\left(1-\mu_{2}\right) y(n \tau)$, and $t=(n+l) \tau, x\left((n+l) \tau^{+}\right)=x((n+l) \tau)+$ $\mu, \mu \geq 0$. We can easily have

Lemma 3.2. Suppose $z(t)$ is a solution of system (2.2) with $z\left(0^{+}\right) \geq 0$, then $z(t) \geq 0$ for $t \geq 0$. and further $z(t)>0 t \geq 0$ for $z\left(0^{+}\right)>0$.

Now, we show that all solutions of (2.3) are uniformly ultimately bounded.

Lemma 3.3. There exists a constant $M>0$ such that $x(t) \leq M, y(t) \leq M$ for each solution $(x(t), y(t))$ of (2.2) with all $t$ large enough. 
Proof. Define $V(t)=k x(t)+y(t)$. When $t \neq n \tau$ and $t \neq(n+l) \tau$, we have

$$
\begin{aligned}
& D^{+} V(t)+d V(t)=k\left[(a+d) x(t)-b x^{2}(t)\right] \\
& \leq-k b\left[x(t)-\frac{k(a+d)}{2 b}\right]^{2}+\frac{k(a+d)^{2}}{4 b} \leq M_{0}
\end{aligned}
$$

where $M_{0}=k(a+d)^{2} / 4 b$. When $t=n \tau, V\left(n \tau^{+}\right)=k x\left(n \tau^{+}\right)+y\left(n \tau^{+}\right)=\left(1-\mu_{1}\right) k x(n \tau)+$ $\left(1-\mu_{2}\right) y(n \tau) \leq k x(n \tau)+y(n \tau)=V(n \tau)$. When $t=(n+l) \tau, V\left((n+l) \tau^{+}\right)=k x\left((n+l) \tau^{+}\right)+y$ $\left((n+l) \tau^{+}\right)=k x((n+l) \tau)+\mu+y((n+l) \tau)=V((n+l) \tau)+\mu$. From ([[6], Lemma 2.2, p. 23]), for $t \in(n \tau,(n+l) \tau]$ and $((n+l) \tau,(n+1) \tau]$, we have

$$
\begin{gathered}
V(t) \leq V\left(0^{+}\right) e^{-\mathrm{d} t}+\frac{M_{0}}{d}\left(1-e^{-\mathrm{d} \tau}\right)+\mu \frac{e^{-\mathrm{d}(t-\tau)}}{1-e^{\mathrm{d} \tau}}+\mu \frac{e^{-\mathrm{d} \tau}}{e^{\mathrm{d} \tau}-1} \\
\rightarrow \frac{M_{0}}{d}+\mu \frac{e^{\mathrm{d} \tau}}{e^{\mathrm{d} \tau}-1}, \quad \text { as } t \rightarrow \infty .
\end{gathered}
$$

So $V(t)$ is uniformly ultimately bounded. Hence, by the definition of $V(t)$, there exists a constant $M>0$ such that $x(t) \leq M, y(t) \leq M$ for $t$ large enough. The proof is complete.

If $y(t)=0$, we obtain the subsystem of system (2.2)

$$
\left\{\begin{array}{l}
\frac{\mathrm{d} x(t)}{\mathrm{d} t}=x(t)(a-b x(t)), \quad t \neq(n+l) \tau, \quad t \neq(n+1) \tau, \\
\Delta x\left(t^{+}\right)=-\mu_{1} x(t), \quad t=(n+l) \tau, n \in Z^{+}, \\
\Delta x\left(t^{+}\right)=\mu, \quad t=(n+1) \tau, n \in Z^{+}, \\
x\left(0^{+}\right)=x(0)>0 .
\end{array}\right.
$$

It is easy to solve the first equation of system (3.1) between pulses

$$
x(t)= \begin{cases}\frac{a e^{a(t-n \tau)} x\left(n \tau^{+}\right)}{a+b\left[e^{a(t-n \tau)}-1\right] x\left(n \tau^{+}\right)}, & t \in(n \tau,(n+l) \tau], \\ \frac{a e^{a(t-(n+l) \tau)} x\left((n+l) \tau^{+}\right)}{a+b\left[e^{a(t-(n+l) \tau)}-1\right] x\left((n+l) \tau^{+}\right)}, & t \in((n+l) \tau,(n+1) \tau] .\end{cases}
$$

By considering the last two equations of system (3.1), we obtain the following stroboscopic map of system (3.1):

$$
x\left((n+1) \tau^{+}\right)=\frac{\left(1-\mu_{1}\right) a e^{a \tau} x\left(n \tau^{+}\right)}{a+b e^{a l \tau}\left[1+\left(1-\mu_{1}\right)\left(e^{a(1-l) \tau}-1\right)\right] x\left(n \tau^{+}\right)}+\mu .
$$

Taking $A=\left(1-\mu_{1}\right) a e^{a \tau}>0$ and $B=b e^{a l \tau}\left[1+\left(1-\mu_{1}\right)\left(e^{a(1-l) \tau}-1\right)\right]>0$, we can rewrite (3.3) as

$$
x\left((n+1) \tau^{+}\right)=\frac{A x\left(n \tau^{+}\right)}{a+B x\left(n \tau^{+}\right)}+\mu .
$$

Referring to [11], we can easily prove that (3.4) has unique positive fixed point

$$
x^{*}=\frac{(A+\mu B-a)+\sqrt{(A+\mu B-a)^{2}+4 \mu a B}}{2 B},
$$

which can be easily proved to be globally asymptotically stable.

Then, we can derive the following lemma: 
Lemma 3.4. System (3.1) has a positive periodic solution $\widetilde{x(t)}$. For every solution $x(t)$ of system (3.1), we have $x(t) \rightarrow \widetilde{x(t)}$ as $t \rightarrow \infty$, where

$$
\widetilde{x(t)}=\left\{\begin{array}{l}
\frac{a e^{a(t-n \tau)} x^{*}}{a+b\left[e^{a(t-n \tau)}-1\right] x^{*}}, \quad t \in(n \tau,(n+l) \tau], \\
\frac{\left(1-\mu_{1}\right) a e^{a(t-n \tau)} x^{*}}{a+b\left[\left(e^{a l \tau}-1\right)+\left(1-\mu_{1}\right)\left(e^{a(t-n \tau)}-e^{a l \tau}\right)\right] x^{*}}, \quad t \in((n+l) \tau,(n+1) \tau] .
\end{array}\right.
$$

\section{The dynamics}

In this article, we will prove that the predator-extinction periodic solution is globally asymptotically stable and system (2.2) is permanent.

\subsection{The extinction}

From above discussion, we know that (2.2) has a predator-extinction periodic solution $(\widetilde{x(t)}, 0)$. Then we have following theorem.

Theorem 4.1. If

$$
\ln \frac{1}{1-\mu_{1}}>a \tau-2\left[\ln \left(1+\frac{b\left(\mathrm{e}^{a l \tau}-1\right) x^{*}}{a}\right)+\ln \left(1+\frac{b\left(1-\mu_{1}\right)\left(\mathrm{e}^{a \tau}-\mathrm{e}^{a l \tau}\right) x^{*}}{a+b\left(\mathrm{e}^{a l \tau}-1\right)}\right)\right],
$$

and

$$
\ln \frac{1}{1-\mu_{2}}>\frac{k \beta}{b}\left[\ln \left(1+\frac{b\left(e^{a l \tau}-1\right) x^{*}}{a}\right)+\ln \left(1+\frac{b\left(1-\mu_{1}\right)\left(e^{a \tau}-e^{a l \tau}\right) x^{*}}{a+b\left(e^{a l \tau}-1\right)}\right)\right]-\mathrm{d} \tau,
$$

hold, then predator-extinction periodic solution $(\widetilde{x(t)}, 0)$ of $(2.2)$ is globally asymptotically stable. Where $x^{*}$ is defined as (3.5):

Proof. First, we prove the local stability. Define $x_{1}(t)=x(t)-\widetilde{x(t)}, y(t)=y(t)$, we have the following linearly similar system of system (2.2):

$$
\left(\begin{array}{c}
\frac{\mathrm{d} x_{1}(t)}{\mathrm{d} t} \\
\frac{\mathrm{d} y(t)}{\mathrm{d} t}
\end{array}\right)=\left(\begin{array}{cc}
a-2 b \widetilde{x(t)} & -\beta \widetilde{x(t)} \\
0 & -d
\end{array}\right)\left(\begin{array}{c}
x_{1}(t) \\
y(t)
\end{array}\right) .
$$

It is easy to obtain the fundamental solution matrix

$$
\Phi(t)=\left(\begin{array}{cc}
\left.\exp \left(\int_{0}^{t}(a-2 b \widetilde{x(s})\right) \mathrm{d} s\right) & * \\
0 & \exp (-\mathrm{d} t)
\end{array}\right) .
$$

There is no need to calculate the exact form of $(*)$ as it is not required in the following analysis. The linearization of the third and fourth equations of (2.2) is

$$
\left(\begin{array}{c}
x_{1}\left((n+l) \tau^{+}\right) \\
y\left((n+l) \tau^{+}\right)
\end{array}\right)=\left(\begin{array}{cc}
1-\mu_{1} & 0 \\
0 & 1-\mu_{2}
\end{array}\right)\left(\begin{array}{c}
x_{1}((n+l) \tau) \\
y((n+l) \tau)
\end{array}\right) .
$$

The linearization of the fifth and sixth equations of (2.2) is

$$
\left(\begin{array}{c}
x_{1}\left((n+1) \tau^{+}\right) \\
y\left((n+1) \tau^{+}\right)
\end{array}\right)=\left(\begin{array}{ll}
1 & 0 \\
0 & 1
\end{array}\right)\left(\begin{array}{c}
x_{1}((n+1) \tau) \\
y((n+1) \tau)
\end{array}\right)
$$


The stability of the periodic solution $(\widetilde{x(t)}, 0)$ is determined by the eigenvalues of

$$
M=\left(\begin{array}{cc}
1-\mu_{1} & 0 \\
0 & 1-\mu_{2}
\end{array}\right)\left(\begin{array}{ll}
1 & 0 \\
0 & 1
\end{array}\right) \Phi(\tau)
$$

which are

$$
\lambda_{1}=\left(1-\mu_{2}\right) \exp (-d \tau)<1, \lambda_{2}=\left(1-\mu_{1}\right) \exp \left(\int_{0}^{\tau}(a-2 b \widetilde{x(s)}) d s\right),
$$

According to the Floquet theory [6], if $\left|\lambda_{2}\right|<1$, i.e. (4.1) holds, then $(\widetilde{x(t)}, 0)$ is locally stable.

The following study is to prove the global attraction, choose $\varepsilon>0$ such that

$$
\rho=\left(1-\mu_{2}\right) \exp \left(\int_{0}^{\tau}[k \beta(\widetilde{x(t)}+\varepsilon)-d] \mathrm{d} t\right)<1,
$$

From the first equation of (2.2), we notice that $\mathrm{d} x(t) / \mathrm{d} t \leq x(t)(a-b x(t))$, so we consider following impulsive differential equation

$$
\left\{\begin{array}{l}
\frac{\mathrm{d} z(t)}{\mathrm{d} t}=z(t)(a-b z(t)), \quad t \neq(n+l) \tau, \quad t \neq(n+1) \tau \\
\Delta z(t)=-\mu_{1} z(t), \quad t=(n+l) \tau \\
\Delta z(t)=\mu, \quad t=(n+1) \tau \\
z\left(0^{+}\right)=z\left(0^{+}\right)
\end{array}\right.
$$

From Lemma 3.4 and comparison theorem of impulsive equation (see [[6], Theorem 3.1.1]), we have $x(t) \leq z(t)$ and $z(t) \rightarrow \widetilde{z(t)}$ as $t \rightarrow \infty$, that is

$$
x(t) \leq z(t) \leq \widetilde{x(t)}+\varepsilon,
$$

for all $t$ large enough, for convenience, we may assume (4.2) hold for all $t \geq 0$. From (2.2) and (4.5), we get.

$$
\left\{\begin{array}{l}
\frac{\mathrm{d} y(t)}{\mathrm{d} t} \leq(k \beta(\widetilde{x(t)}+\varepsilon)-d) y(t), \quad t \neq(n+l) \tau, t \neq(n+1) \tau, \\
\Delta y\left(t^{+}\right)=-\mu_{2} y(t), \quad t=(n+l) \tau, n=1,2, \ldots \\
\Delta y\left(t^{+}\right)=0, \quad t=(n+1) \tau, n=1,2, \ldots
\end{array}\right.
$$

So $y\left((n+l+1) \tau^{+}\right) \leq y\left((n+l) \tau^{+}\right)\left(1-\mu_{2}\right) \exp \left[\int_{(n+l) \tau}^{(n+l+1) \tau}(k \beta(\widetilde{x(s)}+\varepsilon)-d) \mathrm{d} s\right]$, hence $y((n$ $\left.+l) \tau^{+}\right) \leq y\left(l \tau^{+}\right) \rho^{n}$ and $y\left((n+l) \tau^{+}\right) \rightarrow 0$ as $n \rightarrow \infty$. Since $0<y(t) \leq y\left((n+l) \tau^{+}\right)\left(1-\mu_{1}\right)$ $e^{\frac{a e^{a l \tau} x^{*}}{a+b\left(e^{a l \tau}-1\right) x^{*}} l \tau+\frac{a\left(1-\mu_{2}\right) e^{a \tau} x^{*}}{\left.a+b\left[\left(e^{a l \tau}-1\right)+e^{a \tau}-e^{a l \tau}\right)\right] x^{*}}(1-l) \tau}$ for $(n+l) \tau<t \leq(n+l+1) \tau$, therefore $y(t) \rightarrow 0$ as $t \rightarrow \infty$.

Next we prove that $x(t) \rightarrow \widetilde{x(t)}$ as $t \rightarrow \infty$. For $\varepsilon>0$, there must exist a $t_{0}>0 \mathrm{such}$ that $0<y(t)<\varepsilon$ for all $t \geq t_{0}$. Without loss of generality, we assume that $0<y(t)<\varepsilon$ for all $t \geq 0$, then, for the first equation of system (2.2), we have

$$
x(t)[(a-\beta \varepsilon)-b x(t)] \leq \frac{\mathrm{d} x(t)}{\mathrm{d} t} \leq x(t)(a-b x(t)),
$$


then, we have $z_{1}(t) \leq x(t) \leq z_{2}(t)$, and $z_{1}(t) \rightarrow \widetilde{x(t)}, z_{2}(t) \rightarrow \widetilde{x(t)}$, as $t \rightarrow \infty$. While $z_{1}$ $(t)$ and $z_{2}(t)$ are the solutions of

$$
\left\{\begin{array}{l}
\frac{\mathrm{d} z_{1}(t)}{\mathrm{d} t}=z_{1}(t)\left[(a-\beta \varepsilon)-b z_{1}(t)\right], \quad t \neq(n+l) \tau, \quad t \neq(n+1) \tau \\
\Delta z_{1}\left(t^{+}\right)=-\mu_{1} z_{1}(t), \quad t=(n+l) \tau, \\
\Delta z_{1}\left(t^{+}\right)=\mu, \quad t=(n+1) \tau \\
z_{1}\left(0^{+}\right)=x\left(0^{+}\right)
\end{array}\right.
$$

and

$$
\left\{\begin{array}{l}
\frac{\mathrm{d} z_{2}(t)}{\mathrm{d} t}=z_{2}(t)\left[a-b z_{2}(t)\right], \quad t \neq(n+l) \tau, \quad t \neq(n+1) \tau \\
\Delta z_{2}\left(t^{+}\right)=-\mu_{2} z_{2}(t), \quad t=(n+l) \tau \\
\Delta z_{2}\left(t^{+}\right)=\mu, \quad t=(n+1) \tau \\
z_{2}\left(0^{+}\right)=I\left(0^{+}\right)
\end{array}\right.
$$

respectively. And

$$
\widetilde{z_{1}(t)} \widetilde{=}\left\{\begin{array}{l}
\frac{(a-\beta \varepsilon) e^{(a-\beta \varepsilon)(t-n \tau)} z_{1}^{*}}{(a-\beta \varepsilon)+b\left[e^{(a-\beta \varepsilon)(t-n \tau)}-1\right] z_{1}^{*},} t \in(n \tau,(n+l) \tau], \\
\frac{\left(1-\mu_{1}\right)(a-\beta \varepsilon) e^{(a-\beta \varepsilon)(t-n \tau)} z_{1}^{*}}{(a-\beta \varepsilon)+b\left[\left(e^{(a-\beta \varepsilon) l \tau}-1\right)+\left(1-\mu_{1}\right)\left(e^{(a-\beta \varepsilon)(t-n \tau)}-e^{(a-\beta \varepsilon) l \tau}\right)\right] z_{1}^{*}}, t \in((n+l) \tau,(n+1) \tau] .
\end{array}\right.
$$

where

$$
z_{1}^{*}=\frac{\left(A_{1}+\mu B_{1}-(a-\beta \varepsilon)\right)+\sqrt{\left(A_{1}+\mu B_{1}-(a-\beta \varepsilon)\right)^{2}+4 \mu(a-\beta \varepsilon) B_{1}}}{2 B_{1}},
$$

and $A_{1}=\left(1-\mu_{1}\right)(a-\beta \varepsilon) e^{(a-\beta \varepsilon) \tau}>0$ and $B_{1}=b e^{(a-\beta \varepsilon) l \tau}\left[1+\left(1-\mu_{1}\right)\left(e^{(a-\beta \varepsilon)(1-l) \tau}-1\right)\right]>0$. Therefore, for any $\varepsilon_{1}>0$. there exists a $t_{1}, t>t_{1}$ such that

$$
\widetilde{z_{1}(t)}-\varepsilon_{1}<x(t)<\widetilde{z_{2}(t)}+\varepsilon,
$$

Let $\varepsilon \rightarrow 0$, so we have

$$
\widetilde{x(t)}-\varepsilon_{1}<x(t)<\widetilde{x(t)}+\varepsilon_{1}
$$

for $t$ large enough, which implies $x(t) \rightarrow \widetilde{x(t)}$ as $t \rightarrow \infty$. This completes the proof.

\subsection{The permanence}

The following study is to investigate the permanence of system (2.2). Before starting this study, we should give the following definition.

Definition 4.2. System (2.2) is said to be permanent if there are constants $m, M>0$ (independent of initial value) and a finite time $T_{0}$ such that for all solutions $(x(t), y(t))$ with all initial values $x\left(0^{+}\right)>0, y\left(0^{+}\right)>0, m \leq x(t) \leq M, m \leq y(t) \leq M$ holds for all $t \geq$ $T_{0}$. Here $T_{0}$ may depends on the initial values $\left(x\left(0^{+}\right),\left(y\left(0^{+}\right)\right)\right.$. 
Theorem 4.3. If

$$
\begin{aligned}
\ln & \frac{1}{1-\mu_{2}} \\
& <\frac{k \beta}{b}\left[\ln \left(1+\frac{b\left(e^{a l \tau}-1\right) x^{*}}{a}\right)+\ln \left(1+\frac{b\left(1-\mu_{1}\right)\left(e^{a \tau}-e^{a l \tau}\right) x^{*}}{a+b\left(e^{a l \tau}-1\right)}\right)\right]-\mathrm{d} \tau,
\end{aligned}
$$

holds, then, system (2.2) is permanent. Where $x^{*}$ is defined as (3.5).

Proof. Let $(x(t), y(t))$ be a solution of (2.2) with $x(0)>0, y(0)>0$. By Lemma 3.3, we have proved there exists a constant $M>0(\beta M<a)$ such that $x(t) \leq M, y(t) \leq M$ for $t$ large enough. We may assume $x(t) \leq M, y(t) \leq M$ for $t \geq 0$.

In this view, for the first equation of system (2.2), we have

$$
\frac{\mathrm{d} x(t)}{\mathrm{d} t}>x(t)[(a-\beta M)-b x(t)],
$$

then, we obtain the following comparative impulsive differential equation

$$
\left\{\begin{array}{l}
\frac{\mathrm{d} n(t)}{\mathrm{d} t}=n(t)[(a-\beta M)-b n(t)], \quad t \neq(n+l) \tau, \quad t \neq(n+1) \tau, \\
\Delta n\left(t^{+}\right)=-\mu_{1} n(t), \quad t=(n+l) \tau, \\
\Delta n\left(t^{+}\right)=\mu, \quad t=(n+1) \tau, \\
n\left(0^{+}\right)=x\left(0^{+}\right) .
\end{array}\right.
$$

Analyzing (4.14) with similarity as (4.8), we have $n(t) \rightarrow \widetilde{n(t)}$, and

$$
\widetilde{n(t)}=\left\{\begin{array}{l}
\frac{(a-\beta M) e^{(a-\beta M)(t-n \tau)} n^{*}}{(a-\beta M)+b\left[e^{(a-\beta M)(t-n \tau)}-1\right] n^{*}}, t \in(n \tau,(n+l) \tau], \\
\frac{\left.\left(1-\mu_{1}\right)(a-\beta M) e^{(a-\beta M)}\right)^{(t-n \tau)} n^{*}}{(a-\beta M)+b\left[\left(e^{(a-\beta M) l \tau}-1\right)+\left(1-\mu_{1}\right)\left(e^{(a-\beta M)(t-n \tau)}-e^{(a-\beta M) l \tau}\right)\right] n^{*}}, t \in((n+l) \tau,(n+1) \tau],
\end{array}\right.
$$

where

$$
n^{*}=\frac{\left(A_{2}+\mu B_{2}-(a-\beta M)\right)+\sqrt{\left(A_{2}+\mu B_{2}-(a-\beta M)\right)^{2}+4 \mu(a-\beta M) B_{2}}}{2 B_{2}}
$$

and $A_{2}=\left(1-\mu_{1}\right)(a-\beta M) e^{(a-\beta M) \tau}>0$ and $B_{2}=b e^{(a-\beta M) l \tau}\left[1+\left(1-\mu_{1}\right)\left(e^{(a-\beta M)(1-l) \tau}\right.\right.$. $1)]>0$. Following comparative theory of impulsive differential equation [6], we know there exists a $\varepsilon_{2}$ such that $x(t)>\widetilde{n(t)}-\varepsilon_{2}$ for all $t$ large enough, and $\varepsilon_{2}>0$. So $x(t)>\left[n^{*}\right.$ $\left.+\left(1-\mu_{1}\right)(a-\beta M) e^{(a-\beta M) l \tau} /(a-\beta M)+b\left(e^{(a-\beta M) l \tau}-1\right)\right]-\varepsilon_{2}=m_{2}$ for $t$ large enough. Thus, we only need to find $m_{1}>0$ such that $y(t) \geq m_{1}$ for $t$ large enough. We will do it in the following two steps.

(1) By the condition of Theorem 2, we can select $m_{3}>0, \varepsilon_{1}>0$ small enough such that $0<m_{3}<\frac{a}{\beta}$, and

$$
\sigma=\frac{k \beta}{b}\left[\ln \left(1+\frac{b\left(e^{\left(a-\beta m_{3}\right) l \tau}-1\right) z^{*}}{a-\beta m_{3}}\right)+\ln \left(1+\frac{b\left(1-\mu_{1}\right)\left(e^{\left(a-\beta m_{3}\right) \tau}-e^{\left(a-\beta m_{3}\right) l \tau}\right) z^{*}}{a-\beta m_{3}+b\left(e^{a l \tau}-1\right)}\right)\right]-k \beta \varepsilon_{1} \tau-\mathrm{d} \tau>0 .
$$

where $z^{*}$ is defined as (4.20). We will prove that $y(t)<m_{3}$ cannot hold for $t \geq 0$. 
Otherwise,

$$
\left\{\begin{array}{l}
\frac{\mathrm{d} x(t)}{\mathrm{d} t} \geq x(t)\left[\left(a-\beta m_{3}\right)-b x(t)\right], \quad t \neq(n+l) \tau, \quad t \neq(n+1) \tau, \\
\Delta x(t)=-\mu_{1} x(t), \quad t=(n+l) \tau, \\
\Delta x(t)=x(t)+\mu, \quad t=(n+1) \tau,
\end{array}\right.
$$

By Lemma 3.4, we have $x(t) \geq z(t)$ and $z(t) \rightarrow \overline{z(t)}, t \rightarrow \infty$, where $z(t)$ is the solution of

$$
\left\{\begin{array}{l}
\frac{\mathrm{d} z(t)}{\mathrm{d} t} \geq z(t)\left[\left(a-\beta m_{3}\right)-b z(t)\right], \quad t \neq(n+l) \tau, \quad t \neq(n+1) \tau, \\
\Delta z(t)=-\mu_{1} z(t), \quad t=(n+l) \tau, \\
\Delta z(t)=z(t)+\mu, \quad t=(n+1) \tau,
\end{array}\right.
$$

and

$$
\overline{z(t)}=\left\{\begin{array}{l}
\frac{(a-\beta M) e^{\left(a-\beta m_{3}\right)(t-n \tau)} n^{*}}{\left(a-\beta m_{3}\right)+b\left[e^{\left(a-\beta m_{3}\right)(t-n \tau)}-1\right] z^{*}}, t \in(n \tau,(n+l) \tau], \\
\frac{\left(1-\mu_{1}\right)\left(a-\beta m_{3}\right) e^{\left(a-\beta m_{3}\right)(t-n \tau)} z^{*}}{\left(a-\beta m_{3}\right)+b\left[\left(e^{\left(a-\beta m_{3}\right) l \tau}-1\right)+\left(1-\mu_{1}\right)\left(e^{\left(a-\beta m_{3}\right)(t-n \tau)}-e^{\left(a-\beta m_{3}\right) l \tau}\right)\right] z^{*^{\prime}}}, t \in((n+l) \tau,(n+1) \tau],
\end{array}\right.
$$

where

$$
z^{*}=\frac{\left(A_{3}+\mu B_{3}-\left(a-\beta m_{3}\right)\right)+\sqrt{\left(A_{3}+\mu B_{3}-\left(a-\beta m_{3}\right)\right)^{2}+4 \mu\left(a-\beta m_{3}\right) B_{3}}}{2 B_{3}},
$$

and

$$
A_{3}=\left(1-\mu_{1}\right)\left(a-\beta m_{3}\right) e^{\left(a-\beta m_{3}\right) \tau}>0
$$

and

$B_{3}=b e^{\left(a-\beta m_{3}\right) l \tau}\left[1+\left(1-\mu_{1}\right)\left(e^{\left(a-\beta m_{3}\right)(1-l) \tau}-1\right)\right]>0$.

Therefore, there exists a $T_{1}>0$ such that

$$
x(t) \geq z(t) \geq \overline{z(t)}-\varepsilon_{1},
$$

and

$$
\left\{\begin{array}{l}
\frac{\mathrm{d} y(t)}{\mathrm{d} t} \geq \gamma(t)\left[k \beta\left(\overline{z(t)}-\varepsilon_{1}\right)-d\right], \quad t \neq(n+l) \tau \\
\Delta y(t)=-\mu_{2} \gamma(t), \quad t=(n+l) \tau, \quad n=1,2, \ldots
\end{array}\right.
$$

for $t \geq T_{1}$. Let $N_{1} \in N$ and $N_{1} \tau>T_{1}$, integrating (4.21) on $((n+l-1) \tau,(n+l) \tau), n \geq$ $N_{1}$, we have

$$
\gamma((n+l) \tau) \geq\left(1-\mu_{2}\right) y((n+l-1) \tau) \exp \left(\int_{(n+l-1) \tau}^{(n+l) \tau}\left[k \beta\left(\overline{z(t)}-\varepsilon_{1}\right)-d\right] \mathrm{d} t\right)=\left(1-\mu_{2}\right) y((n+l-1) \tau) e^{\sigma},
$$

then, $y\left(\left(N_{1}+k+l\right) \tau\right) \geq\left(1-\mu_{2}\right)^{k} y\left(\left(N_{1}+l\right) \tau\right) e^{k \sigma} \rightarrow \infty$, as $k \rightarrow \infty$, which is a contradiction to the boundedness of $y(t)$. Hence there exists a $t_{1}>0$ such that $y(t) \geq m_{3}$.

(2) If $y(t) \geq m_{3}$ for $t \geq t_{1}$, then our aim is obtained. Hence, we only need to consider those solutions which leave region $R=\left\{(x(t), y(t)) \in R_{+}^{2}: \gamma(t)<m_{3}\right\}$ and reenter it again. Let $t^{*}=\inf _{t \geq t_{1}}\left\{\gamma(t)<m_{3}\right\}$, there are two possible cases for $t^{*}$.

Case 1. $t^{*}=(n+l-1) \tau, n_{1} \in Z_{+}$, then $x(t) \geq m_{3}$ for $t \in\left[t_{1}, t^{*}\right)$ and $y\left(t^{*}\right)=m_{3}$, and $y$ $\left(t^{*+}\right)=y\left(t^{*}\right) \leq m_{3}$. Select $n_{2}, n_{3} \in N$, such that 


$$
\begin{gathered}
\left(n_{2}-1\right) \tau>T_{2}=\frac{\ln \left(\frac{\varepsilon_{1}}{M}\right)}{k \beta m_{3}-d}, \\
\left(1-\mu_{2}\right)^{n_{2}} e^{n_{3} \sigma} e^{n_{2} \sigma_{1} \tau}>\left(1-\mu_{2}\right)^{n_{2}} e^{n_{3} \sigma} e^{\left(n_{2}+1\right) \sigma_{1} \tau}>1,
\end{gathered}
$$

where $\sigma_{1}=k \beta m_{2}-d<0$, Let $T=n_{1} \tau+n_{2} \tau$. We claim that there must be a $t_{2} \in\left[t^{*}\right.$, $\left.t^{*}+T\right]$ such that $y\left(t_{2}\right)>m_{3}$, otherwise, consider (4.21) with $z\left(t^{*+}\right)=y\left(t^{*+}\right)$. We have

$$
z(t)=z\left(n_{1}^{+}\right) e^{\left(k \beta m_{3}-d\right) t}+\overline{z(t)}, \quad t \in((n-1) \tau, \quad(n+l-1) \tau],
$$

and $n_{1}+1 \leq n \leq n_{2}+n_{3}$, then

$$
|z(t)-\overline{z(t)}|<M e^{\left(k \beta m_{3}-d\right)\left(t-n_{1} \tau\right)}<\varepsilon_{1},
$$

and $\gamma(t) \leq z(t) \leq \overline{z(t)}+\varepsilon_{1},\left(n_{1}+n_{2}-1\right) \tau \leq t \leq t^{*}+T$, which implies (4.22) holds for $t^{*}+n_{2} \tau \leq t \leq t^{*}+T$. As in step 1 , we have

$$
\left.\gamma\left(t^{*}+T\right) \geq \gamma\left(t^{*}+n_{2}\right) \tau\right) e^{n_{3} \sigma},
$$

The second equation of system (2.1) gives

$$
\left\{\begin{array}{l}
\frac{\mathrm{d} y(t)}{\mathrm{d} t} \geq \gamma(t)\left(k \beta m_{2}-d\right)=\sigma_{1} \gamma(t), \quad t \neq(n+l-1) \tau, \\
\Delta y(t)=-\mu_{2} \gamma(t), \quad t=(n+l-1) \tau,
\end{array}\right.
$$

Integrating (4.23) on $\left[t^{*}, t^{*}+n_{2} \tau\right]$, we have

$$
\gamma\left(t^{*}+n_{2} \tau\right) \geq\left(1-\mu_{2}\right)^{n_{2}} m_{3} e^{\sigma_{1} n_{2} \tau},
$$

thus we have

$$
\gamma\left(t^{*}+T\right) \geq\left(1-\mu_{2}\right)^{n_{2}} m_{3} e^{\sigma_{1} n_{2} \tau} e^{n_{3} \sigma}>m_{3},
$$

which is a contradiction. Let $\bar{t}=\inf _{t \geq t^{*}}\left\{\gamma(t) \geq m_{3}\right\}$, thus $\gamma(\bar{t}) \geq m_{3}$ for $t \in\left[t^{*}, \bar{t}\right]$, we have $\gamma(t) \geq \gamma\left(t^{*}\right) e^{\sigma_{1}\left(t-t^{*}\right)} \geq\left(1-\mu_{2}\right)^{n_{2}+n_{3}} m_{3} e^{\sigma_{1}\left(n_{2}+n_{3}\right)}=m_{1}$ for $t \geq \bar{t}$. So we have $y(t) \geq$ $m_{1}$. The same arguments can be continued since $\gamma(\bar{t}) \geq m_{3}$. Hence $\gamma(t) \geq m_{1}$ for all $t \geq \bar{t}$.

Case 2. $t \neq(n+l-1) \tau, n \in Z_{+}$, then $y(t) \geq m_{3}$ for $t \in\left[t_{1}, t^{*}\right)$, and $y\left(t^{*}\right)=m_{3}$. Suppose $t^{*} \in\left(\left(n_{1}^{\prime}+l-1\right) \tau,\left(n_{1}^{\prime}+l\right) \tau\right),\left(t \in Z_{+}\right)$, then there are two possible cases for $t \in\left(t^{*},\left(n_{1}^{\prime}+l\right) \tau\right)$.

Case 2(a). $y(t) \leq m_{3}$ for all $t \in\left(t^{*},\left(n_{1}^{\prime}\right) \tau\right)$. Similar to case 1., we can prove that there must be a $t_{2}^{\prime} \in\left[\left(n_{1}^{\prime}+l\right) \tau,\left(n_{1}^{\prime}+l\right) \tau+T\right]$ such that $\gamma\left(t_{2}^{\prime}\right)>m_{3}$. Here we omit it.

Let $\bar{t}=\inf _{t>t^{*}}\left\{\gamma(t)>m_{3}\right\}$, then $y(t) \leq m_{3}$ for $t \in\left(t^{*}, \bar{t}\right)$ and $y(\bar{t})=m_{3}$. For $t \in\left(t^{*}, \bar{t}\right)$, we have

$$
\gamma(t) \geq\left(1-p_{1}\right)^{n_{2}+n_{3}} m_{3} e^{\left(n_{2}+n_{3}+1\right) \sigma_{1} \tau} .
$$

Let $m_{1}=\left(1-\mu_{2}\right)^{n_{2}+n_{3}} m_{3} e^{\left(n_{2}+n_{3}+1\right) \sigma_{1} \tau}<m_{1}^{\prime}$, so $y(t) \geq m_{1}$ for $t \in\left(t^{*}, \bar{t}\right)$. For $t>\bar{t}$, the same arguments can be continued since $y(t) \geq m_{1}$.

Case 2(b). There exists a $t \in\left(t^{*},\left(n_{1}^{\prime}\right) \tau\right)$ such that $y(t)>m_{3}$. Let $\hat{t}=\inf _{t>t^{*}}\left\{y(t)>m_{3}\right\}$, then $y(t) \leq m_{3}$ for $t \in\left(t^{*}, \hat{t}\right)$ and $y(\hat{t})=m_{3}$. For $t \in\left(t^{*}, \hat{t}\right)$, (4.23) holds true, integrating (4.23) on $\left(t^{*}, \hat{t}\right)$, we derive

$$
\gamma(t) \geq \gamma\left(t^{*}\right) e^{\sigma_{1}\left(\hat{t}-t^{*}\right)} \geq m_{3} e^{\sigma_{1} \tau}>m_{1}
$$


Since $y(\hat{t}) \geq m_{3}$ for $t>\hat{t}$, the same arguments can be continued. Hence $y(t) \geq m_{3}$ for $t \geq t_{1}$. This completes the proof.

\section{Discussion}

In this article, according to the fact of biological resource management, we proposed and investigated a predator-prey model with impulsive releasing prey population and impulsive harvesting predator population and prey population at different fixed moment. We analyze that the predator-extinction periodic solution of this system is globally asymptotic stability. If it is assumed that $x(0)=2, y(0)=2, a=2, b=1, d=1$, $\beta=0.6, k=0.9, \mu_{1}=0.2, \mu_{2}=0.6, \mu=3, l=0.25, \tau=1$, obviously, the condition of predator-extinction are satisfied, then the predator-extinction periodic solution of system (2.1) is globally asymptotically stable (the numerical simulation can be seen in Figures 1,2 , and 3). We also obtain the condition of the permanence of system (2.2). If it is assumed that $x(0)=2, y(0)=2, a=2, b=1, d=1, \beta=0.6, k=0.9, \mu_{1}=0.2, \mu_{2}=$ $0.4, \mu=3, l=0.25, \tau=1$, obviously, the permanent condition of system (2.2) is satisfied, then, system (2.1) is permanent (the numerical simulation can also be seen in Figures 4, 5, and 6). From results of the numerical simulation, we know that there exists an impulsive harvesting predator population threshold $\mu_{2}^{* *}$, which satisfies $0.4<\mu_{2}^{* *}<0.6$. If $\mu_{2}>\mu_{2}^{* *}$, the predator-extinction periodic solution $(\widetilde{x(t)}, 0)$ of system (2.2) is globally asymptotically stable. If $\mu_{2}<\mu_{2}^{* *}$, system (2.2) is permanent.

From Theorems 4.1 and 4.3, we can easily guess that there must exist an impulsive harvesting predator population threshold $\mu_{2}^{*}$. If $\mu_{2}>\mu_{2}^{*}$, the predator-extinction periodic solution $(\widetilde{x(t)}, 0)$ of system $(2.2)$ is globally asymptotically stable. If $\mu_{2}>\mu_{2}^{*}$,

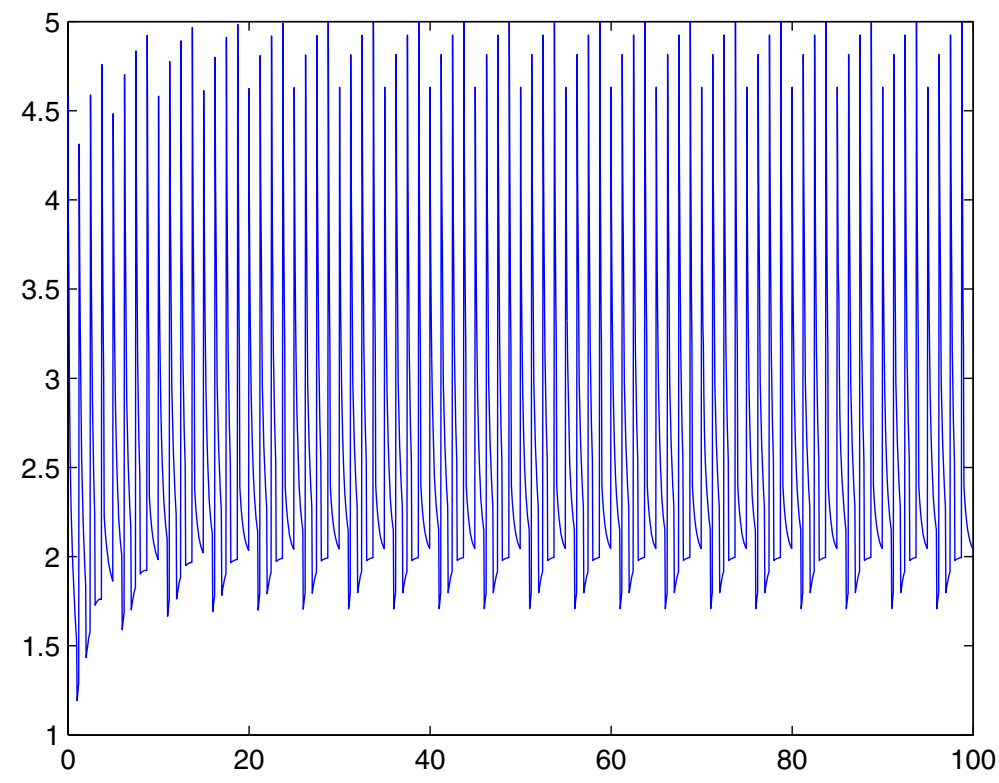

Figure 1 Time-series of $x(t)$ of globally asymptotic stability predator-extinction periodic solution of system (2.2) with $\mathrm{x}(0)=2, \mathrm{y}(0)=2, \mathrm{a}=2, \mathrm{~b}=1, \mathrm{~d}=1, \beta=0.6, \mathrm{k}=0.9, \mu_{1}=0.2, \mu_{2}=0.6, \mu=3$, I $=0.25, \tau=1$. 


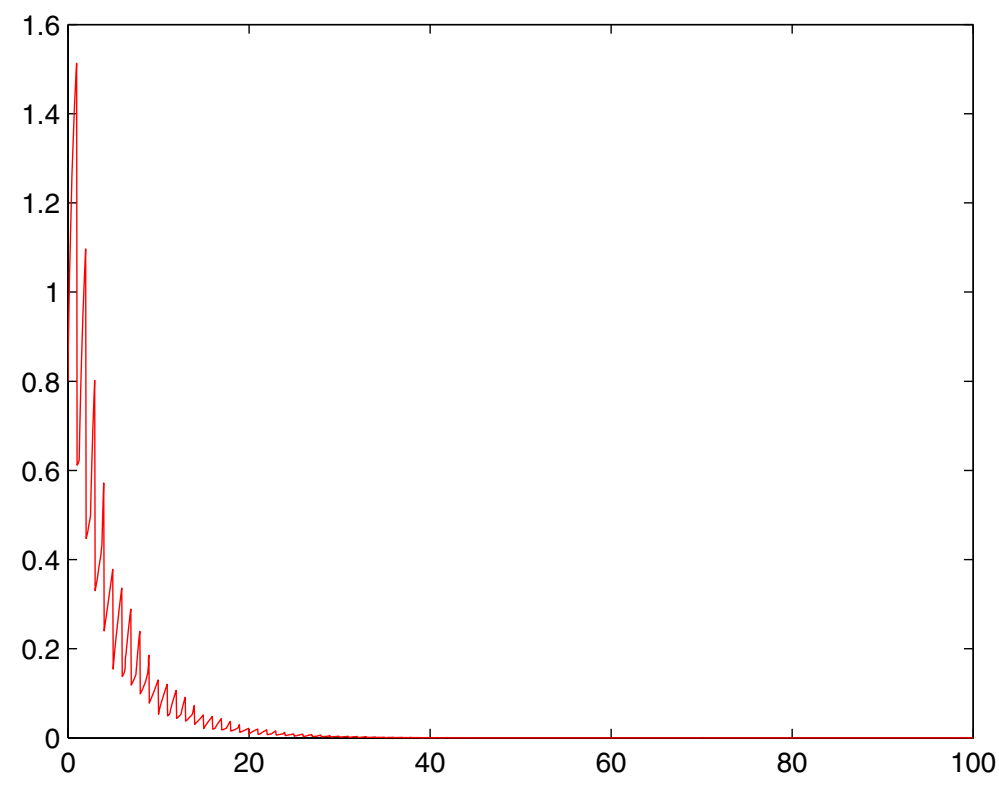

Figure 2 Time-series of $y(t)$ of globally asymptotic stability predator-extinction periodic solution of system (2.2) with $\mathrm{x}(0)=2, \mathrm{y}(0)=2, \mathrm{a}=2, \mathrm{~b}=1, \mathrm{~d}=1, \beta=0.6, \mathrm{k}=0.9, \mu_{1}=0.2, \mu_{2}=0.6, \mu=3$, I $=0.25, \tau=1$.

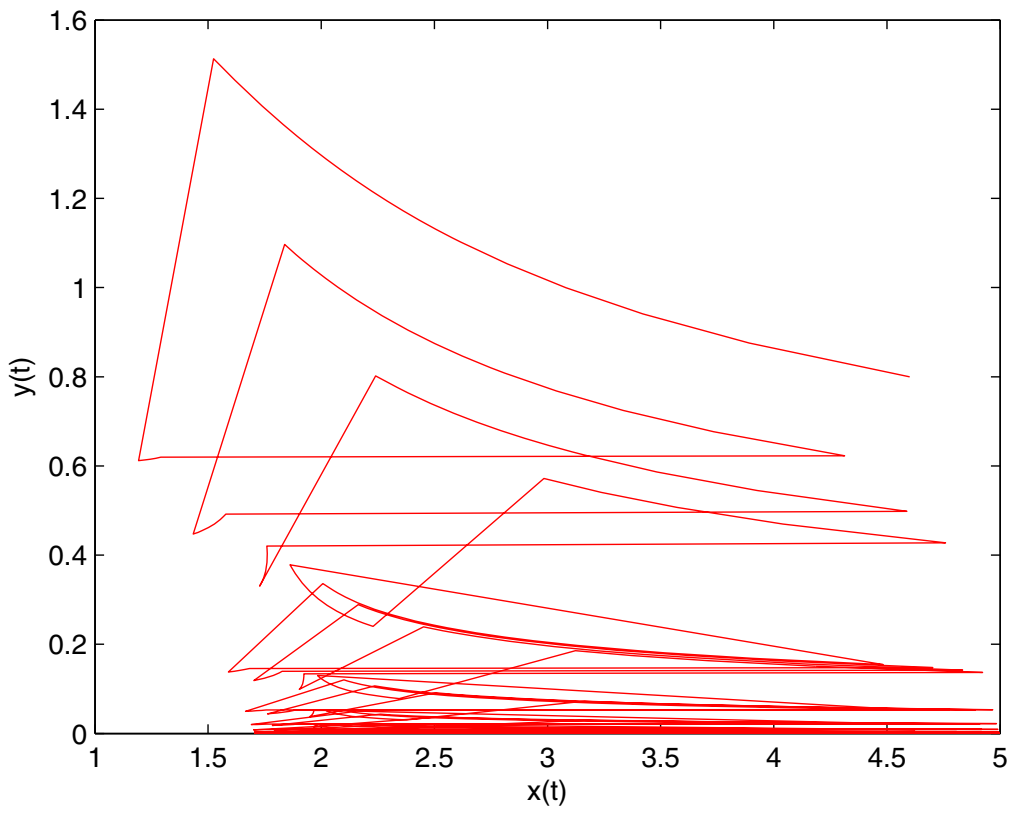

Figure 3 Phase diagram of globally asymptotic stability predator-extinction periodic solution of system (2.2) with $\mathrm{x}(0)=2, \mathrm{y}(0)=2, \mathrm{a}=2, \mathrm{~b}=1, \mathrm{~d}=1, \beta=0.6, \mathrm{k}=0.9, \mu_{1}=0.2, \mu_{2}=0.6, \mu=3$, I $=0.25, \tau=1$. 


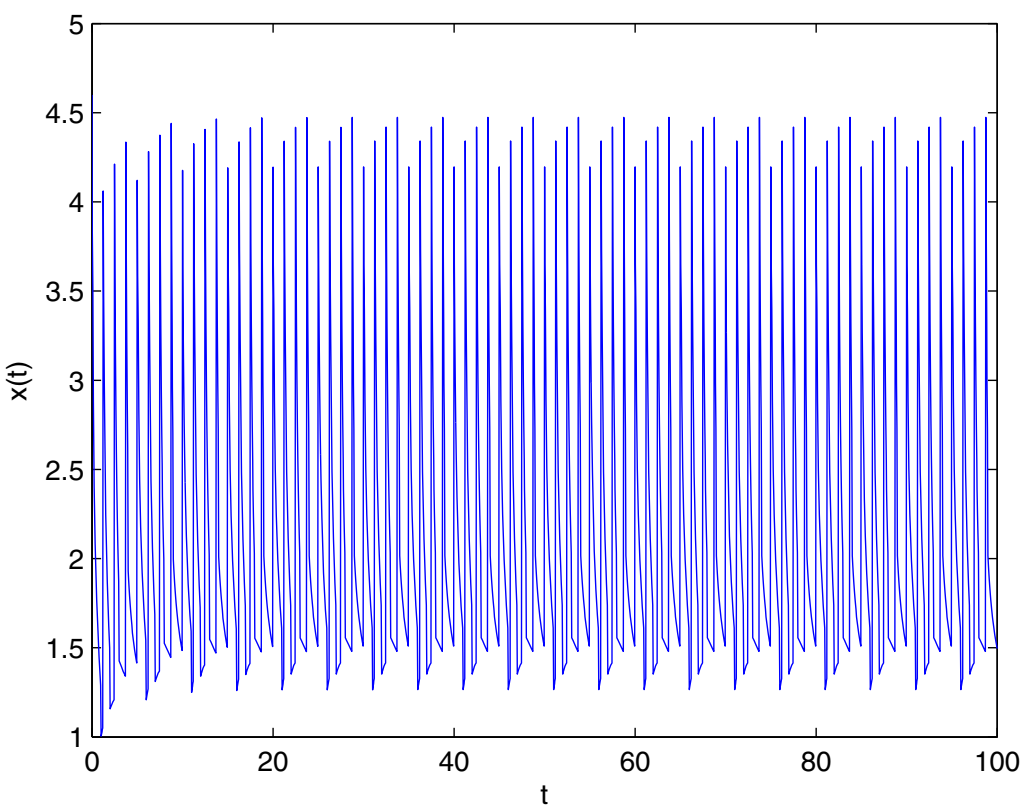

Figure 4 Time-series of $x(t)$ of permanence of system (2.2) with $x(0)=2, y(0)=2, a=2, b=1, d=$ $1, \beta=0.6, \mathrm{k}=0.9, \mu_{1}=0.2, \mu_{2}=0.4, \mu=3, \mathrm{I}=0.25, \tau=1$.

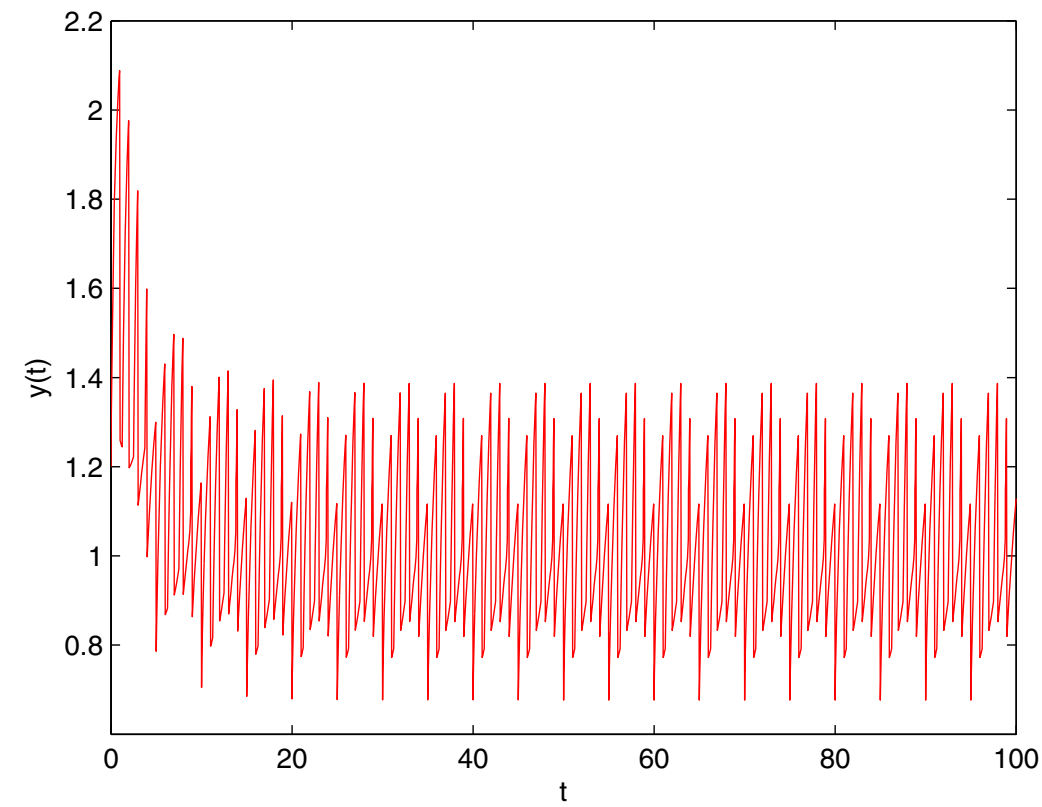

Figure 5 Time-series of $y(t)$ of permanence of system (2.2) with $x(0)=2, y(0)=2, a=2, b=1, d=$ $1, \beta=0.6, \mathrm{k}=0.9, \mu_{1}=0.2, \mu_{2}=0.4, \mu=3, \mathrm{I}=0.25, \tau=1$. 


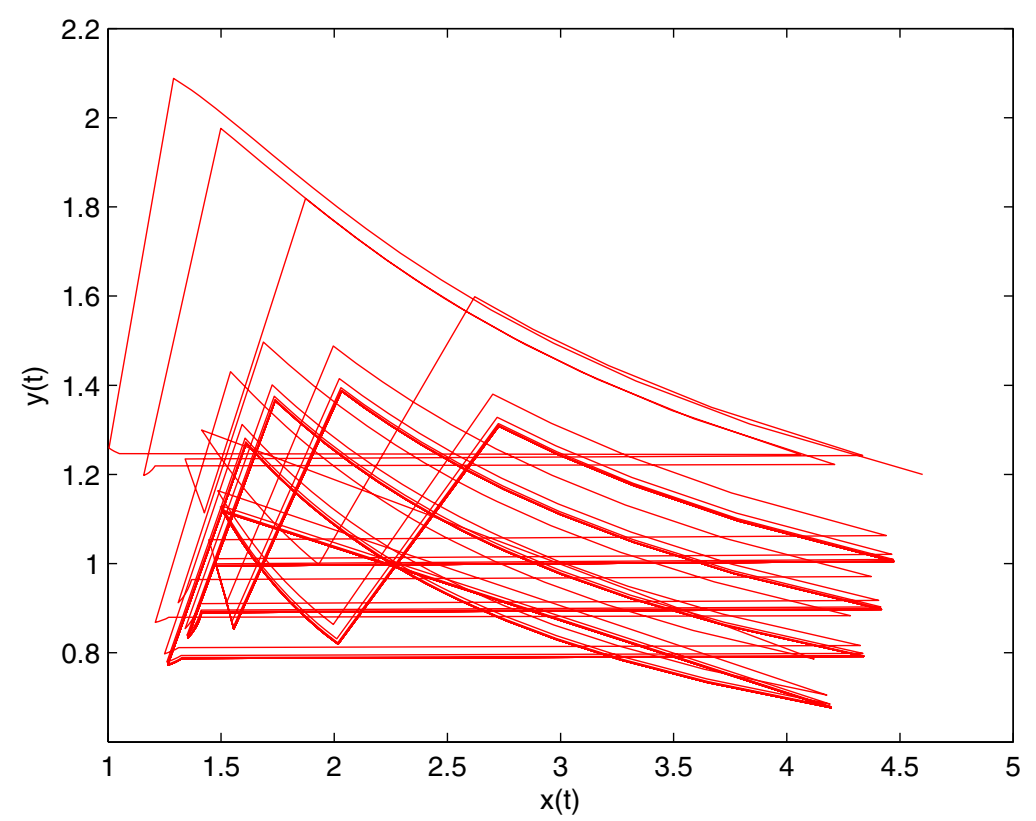

Figure 6 Phase diagram of permanence of system (2.2) with $\mathrm{x}(0)=2, \mathrm{y}(0)=2, \mathrm{a}=2, \mathrm{~b}=1, \mathrm{~d}=1$, $\beta=0.6, \mathrm{k}=0.9, \mu_{1}=0.2, \mu_{2}=0.4, \mu=3, \mathrm{I}=0.25, \tau=1$.

system (2.2) is permanent. The same discussion can be applied to parameters $\mu_{1}$ and $\tau$. These results show that the impulsive effect plays an important role for the permanence of system (2.2). Our results provide reliable tactic basis for the practically biological resource management.

\section{Acknowledgements}

The authors were grateful to the associate editor, Professor Leonid Berezansky, and the referees for their helpful suggestions that are beneficial to our original article. This study was supported by the Development Project of Nature Science Research of Guizhou Province Department (No. 2010027), the National Natural Science Foundation of China (10961008), and the Science Technology Foundation of Guizhou(2010J2130).

\section{Author details}

${ }^{1}$ School of Mathematics and Statistics, Guizhou Key Laboratory of Economic System Simulation, Guizhou University of Finance and Economics, 550004 Guiyang, P. R. China ${ }^{2}$ Institute of Mathematics, Academy of Mathematics and System Sciences, 100080 Beijing, P. R. China

\section{Authors' contributions}

JJ carried out the main part of this article, LC corrected the manuscript. SC brought forward some suggestion on this article. All authors have read and approved the final manuscript.

\section{Competing interests}

The authors declare that they have no competing interests.

Received: 27 August 2011 Accepted: 11 February 2012 Published: 11 February 2012

\section{References}

1. Clark, CW: Mathematical Bioeconomics. Wiley, New York (1990)

2. Goh, BS: Management and Analysis of Biological Populations. Elsevier, Amsterdam (1980)

3. Wang, WD, Chen, LS: A predator-prey system with stage structure for predator. Comput Math Appl. 33(8):83-91 (1997). doi:10.1016/S0898-1221(97)00056-4

4. Lakshmikantham, V, Bainov, DD, Simeonov, P: Theory of Impulsive Differential Equations. World Scientific, Singapore (1989)

5. Song, XY, Chen, LS: Optimal harvesting and stability for a predator-prey system with stage structure. Acta Math Appl (English series). 18(3):423-430 (2002). doi:10.1007/s102550200042 
6. Bainov, D, Simeonov, P: Impulsive Differential Equations: Periodic Solutions and Applications. In Pitman Mongraphs and Surveys in Pure and Applied Mathematics, vol. 66,Wiley, New York (1993)

7. Meng, X, Jiao, J, Chen, L: Global dynamics behaviors for a nonautonomous Lotka- Volterra almost periodic dispersal system with delays. Nonlinear Anal Theory Methods Appl. 68, 3633-3645 (2008). doi:10.1016/j.na.2007.04.006

8. Jiao, J, Chen, L: A pest management SI model with biological and chemical control concern. Appl Math Comput. 183, 1018-1026 (2006). doi:10.1016/j.amc.2006.06.070

9. Meng, X, Chen, L: Permanence and global stability in an impulsive Lotka-Volterra N-species competitive system with both discrete delays and continuous delays. Int J Biomath. 1, 179-196 (2008). doi:10.1142/S1793524508000151

10. Jiao, J, Chen, L: A stage-structured holling mass defence predator-prey model with impulsive perturbations on predators. Appl Math Comput. 189, 1448-1458 (2007). doi:10.1016/j.amc.2006.12.043

11. Meng, $X$, Jiao, J, Chen, L: The dynamics of an age structured predator-prey model with disturbing pulse and time delays. Nonlinear Anal Real World Appl. 9, 547-561 (2008). doi:10.1016/j.nonrwa.2006.12.001

doi:10.1186/1687-1847-2012-9

Cite this article as: Jiao et al:: Dynamical analysis of a biological resource management model with impulsive releasing and harvesting. Advances in Difference Equations 2012 2012:9.

\section{Submit your manuscript to a SpringerOpen ${ }^{\odot}$} journal and benefit from:

Convenient online submission

- Rigorous peer review

- Immediate publication on acceptance

- Open access: articles freely available online

- High visibility within the field

- Retaining the copyright to your article

Submit your next manuscript at $\gg$ springeropen.com 